\title{
Relação entre Percepção de Suporte Familiar e Vulnerabilidade ao Estresse no Trabalho
}

\author{
Relationship Between Perceived Family Support and Vulnerability to Stress at Work \\ Relación Entre la Percepción de Apoyo a La Familia y la Vulnerabilidad al Estrés en \\ el Trabajo \\ Regiane Ribeiro de Aquino \\ Makilim Nunes Baptistal \\ Mayra Silva de Souza \\ Universidade São Francisco
}

\begin{abstract}
Resumo
A família constitui-se como uma das principais formas de suporte social, sendo que quanto maior o suporte que o individuo possui, menor tendência a desenvolver transtornos mentais e ser vulnerável a situações estressoras. Considerando a multiplicidade de papéis que a família tem diante de diversas situações estressoras, inclusive a laboral, o presente artigo investigou a relação entre percepção de suporte familiar e vulnerabilidade ao estresse no trabalho. Para tal, aplicou-se, além de um questionário de identificação a Escala de Vulnerabilidade ao Estresse no Trabalho -EVENT e o Inventário de Percepção de Suporte Familiar - IPSF em 414 universitários com idade média de 25 anos (DP=6,27), sendo 56,8\% mulheres. Os resultados indicaram correlações entre todas as dimensões da EVENT com o IPSF, sendo que este último diferenciou-se estatística e significativamente entre os sexos. Ainda, os resultados demonstraram que quanto maior a carga horária de trabalho, maior vulnerabilidade ao estresse.

Palavras -chave: família; estresse; trabalho.
\end{abstract}

\begin{abstract}
The family constitute itself as a major form of social support, and the greater the support that the individual possesses, the less likely to develop mental disorders and be vulnerable to stressful situations. Considering the multiplicity of roles that the family is faced with many stressful situations, including labor, this paper investigated the relationship between perceived family support and vulnerability to stress at work. To this end, it was applied an identify questionnaire, the Escala de Vulnerabilidade ao Estresse no Trabalho- EVENT and, the Inventário de Percepção de Suporte Familiar - IPSF on 414 university students with an average age of 25 years $(\mathrm{SD}=6.27)$, with $56.8 \%$ women. The results indicated correlations between all dimensions of EVENT with the IPSF, the latter differed statistically significantly between the sexes. The results also showed that a higher workload, greater vulnerability to stress.
\end{abstract}

Key words: family; stress; work.

\section{Resumen}

La familia se estableció como una de las principales formas de apoyo social, siendo que cuanto más apoyo tenga el individuo, menores serán las probabilidades de desarrollar trastornos mentales y ser vulnerable a situaciones de estrés. Teniendo en cuenta la multiplicidad de roles que la familia enfrenta em varias situaciones de estrés, incluyendo el trabajo, este artículo analizó la relación entre el apoyo percibido de la familia y la vulnerabilidad al estrés laboral. Para este fin se aplicó un cuestionario en 414 estudiantes universitarios con una edad promedia de 25 años (DT $=6,27$ ), siendo $56,8 \%$ mujeres. Los resultados indicaron correlaciones entre todas las dimensiones del la EVENT con IPSF, siendo que el IPSF presentó diferencias estadisticamente significativas entre los sexos. Los resultados también mostraron que quanto mayor la carga laboral, mayor la tensión. Palabras claves: familia, estrés, trabajo.

\section{Introdução}

A família é um dos primeiros grupos em que se estabelece a aprendizagem de comportamentos e decisões, de onde se origina a cultura, os valores e as normas sociais (OPS, 2003). Enquanto papel social, a família desempenha funções como prover

1 Endereço:Programa de Pós Graduação Strictu-Sensu em Psicologia Comissão de Pós Graduação. Av Alexandre Rodrigues Barbosa, 45 - Centro, CEP 13251-900, Itatiba-SP. Telefone: (11) 4534-8040. alimentos, abrigo e outras necessidades materiais; oferecer proteção frente às diversidades externas; promover os primeiros laços afetivos; favorecer que a identidade pessoal seja construída a partir da identidade familiar; proporcionar integridade e promover a aquisição de capacidades para o enfrentamento das situações e experiências novas; servir como modelo de papéis sexuais; exercitar a integração de papéis e da responsabilidade social e apoiar a criatividade e iniciativa pessoal, como fomento de aprendizagem (Ackerman, 1978).

Ainda nesse mesmo sentido, Caldas (2003) e 
Carvalho e Almeida (2003) ressaltam a importância da família enquanto provedora de necessidades emocionais e econômicas, visto que permite que o indivíduo se sinta valorizado e compreendido, no intuito de produzir, reunir e distribuir recursos para a satisfação dessas necessidades. A família tem sofrido o impacto de todas as mudanças ocorridas na sociedade, incluindo os aspectos econômicos, políticos, tecnológicos e culturais. Por ser considerado o primeiro grupo do qual o ser humano faz parte, a saúde mental das pessoas e o ambiente familiar experenciado são relacionados com frequência (Ackerman, 1978; Féres- Carneiro 1983).

Ackerman (1978) ressaltou a necessidade do estudo das relações familiares em seu contexto micro e macrosocial e a ambivalência da função exercida pelo grupo familiar. O papel da família pode se relacionar aos aspectos positivos como desenvolvimento, saúde, sentimentos de realização e aos negativos, representados pela enfermidade, fracasso e a não promoção de saúde, ou seja, a família favorece a saúde, mas também pode contribuir para o aparecimento da doença. A importância da família no contexto micro está ligada a sua base para os relacionamentos humanos e, em um aspecto macro, em sua relação com a sociedade e a cultura, pois a interface entre esses dois pontos ressalta as vicissitudes familiares que são valorizadas de diferentes formas pela cultura.

A família possui múltiplos papéis no processo de estresse. Ao ser vista como uma instituição social, a vida familiar pode ser considerada propícia a um clima de tensão. Uma tensão vivida no trabalho, pode se estender ao ambiente familiar e vice-versa, mesmo que a família possa ter o papel de oferecer aos seus membros momentos de tranqüilidade. O lar pode representar para muitas pessoas não apenas o lugar preferido após situações de aflição, mas o único lugar em que receberiam aceitação e suporte (Pearlin, 1982).

Além de oferecer sossego e suporte, a família também pode infligir dor emocional e punição. As mudanças institucionais e estruturais da família contemporânea podem contribuir para as questões de estresse em fatores como urbanização, espaço geográfico, diferenças sociais e econômicas entre os pares, mudanças ou perdas de papéis, crescimento dos filhos e/ou morte do parceiro entre outros. Com o aumento da longevidade, muitas pessoas adiam seu desejo de serem pais e só o realizam após a estabilização profissional. Em alguns casos, o declínio da saúde física dos pais ou doenças debilitadoras em pais de filhos jovens, pode fazê-los assumir o papel de cuidadores, desempenhando responsabilidades, ainda muito jovens, o que pode comprometer sua autonomia futura (Pearlin, 1982).

Alchaer-Alchaer, Bahsas-Bahsas, HernándezNieto e Salinas (1994) destacaram que a família influencia no desenvolvimento de enfermidades, morbidade, mortalidade e também, na recuperação de problemas de saúde. Seu nível de funcionamento poderia ainda, refletir positiva ou negativamente na saúde dos indivíduos. No estudo com 500 habitantes venezuelanos maiores de 13 anos, os resultados demonstraram que os maiores níveis de disfunção familiar estavam relacionados com participantes adolescentes, com enfermidades psicossomáticas, situação sócio-econômica baixa, indivíduos obesos, alcoólatras e com níveis maiores de estresse. Foi destacado também, que o nível de eventos de vida estressantes e de ansiedade apresentaram valor preditivo do funcionamento familiar, ainda que com correlação baixa. Foram encontrados ainda, que participantes com maiores níveis de eventos de vida estressantes, tenderam a produzir disfunção familiar moderada e severa. Embora não tivessem sido encontradas influências no funcionamento familiar em virtude do sexo, estado civil e alguns riscos de saúde, observou-se que, entre os eventos de vida estressantes e o funcionamento familiar, a correlação negativa foi altamente significativa. Como disfunção familiar, destaca-se a maneira de compartilhar decisões, satisfação na família, comunicação, solução de problemas e tempo utilizado juntos. Nesse estudo $50,57 \%$ da amostra referiram má relação com os membros da família.

Cooke, Rossmann, McCubbin e Patterson (1988) consideraram que aqueles que sofrem com fatores estressantes, crises ou transições na vida podem buscar no suporte social e familiar, recursos potenciais para favorecer o bem estar. A ação do suporte tanto poderia ocorrer no indivíduo como na família, sendo necessário destacar que as alterações ocorridas na sociedade envolvem o indivíduo, a interação e a configuração familiar.

Baptista, Baptista e Dias (2001) ao discutirem suporte familiar, apontaram a abrangência do termo e encontraram vários estudos destacando o suporte social proveniente da família ou dirigido a ela, com o objetivo de oferecer auxílio, assistência, expressão afetiva e outros. Em seus estudos bibliográficos, observaram que o suporte familiar e o suporte social teriam efeitos atenuantes sobre a ação dos fatores estressantes do cotidiano. Baptista (2005) ressaltou que o suporte social, mais especificamente o suporte familiar, pode ser considerado como amortecedor do efeito da ação de diversos estressores na vida dos indivíduos.

Segundo Castro, Campero e Hernández (1997) o suporte familiar é um conceito multidimensional, complexo e que se associa com a saúde dos indivíduos, sendo visto também como uma das variáveis que explica as oscilações do impacto do estresse sobre a saúde. Verificou-se que o efeito do suporte é compreendido de duas formas: (1) como efeito direto: que favorece os níveis de saúde independente do nível de estresse e (2) como efeito amortecedor: 
que protege o indivíduo dos efeitos dos eventos de vida estressantes. O fenômeno é complexo, mas se observou que o indivíduo que possui relações de apoio seja na forma de família, igreja, amigos entre outros, com frequência possui melhores condições de saúde física e mental.

Campos (2004) considera que o suporte familiar contribui de maneira significativa para a manutenção e a integridade física e psicológica do indivíduo. Seu efeito é tido como benéfico, no membro da família que o recebe, na medida em que o suporte é percebido como disponível e satisfatório. Essa percepção permite que o indivíduo sinta-se reconhecido, amado, acolhido e participante de uma rede em que há trocas de informações, em que os recursos são partilhados, favorecendo assim bem-estar psicológico.

Nos estudos atuais sobre estresse, os pesquisadores consideram que o desgaste físico e emocional atinge as pessoas quase como uma epidemia da modernidade, refletindo seu impacto na saúde física e mental. O termo estresse ocupa lugar de destaque tanto no senso comum, quanto na imprensa e na comunidade científica, relacionado às dificuldades dos indivíduos frente às situações adversas experenciadas como a violência, os problemas financeiros, questões familiares, no trabalho entre outros (Salmond \& Ropis 2005, Calnan, Wadsworth, May, Smith \& Wainwright, 2004).

Selye (1982) é apontado como o precursor em estudos sobre estresse e, em seus registros apontou que a crise que a humanidade vem enfrentando, não difere muito de situações adversas vividas desde a pré-história. Naquele tempo os problemas enfrentados pela humanidade eram em virtude do frio, do trabalho pesado, do medo de animais ferozes, de fome e doenças. Com o desenvolvimento das sociedades, o homem enfrentou guerras mundiais e grandes mudanças tecnológicas, presentes até a atualidade. No século XXI, os problemas vivenciados são outros, mas permanecem os sentimentos de tensão, insatisfação, pressão e fragilidades sentidas, principalmente no que diz respeito ao estresse e sua relação com trabalho (Myers \& Myers, 2004).

Embora se trate de um construto de grande relevância, vários autores destacaram que não há uma definição amplamente aceita sobre o conceito de estresse, quando ocorre, como observá-lo e quantificálo. As dificuldades de conceituação do construto relacionam-se à variedade de modelos teóricos existentes, pois alguns o caracterizam em termos de respostas e outros o descrevem como um conjunto de estímulos. Há, ainda, modelos que sugerem que o estresse pode ser avaliado com foco no indivíduo, no ambiente ou em ambos (Baum \& Posluszny, 1999; Mota, Franco \& Motta, 1999).

O conceito de estresse como resposta pode ser observado nos estudos de Selye (1982), que o definiu como o conjunto de reações que um organismo desenvolve ao ser submetido a uma situação que exige esforço e adaptação. Diante da ação do estressor, o organismo se prepara para a fuga ou luta e esta ação produzida por agentes nocivos, é chamada de Síndrome Geral de Adaptação, ou seja, a expressão somática em que o corpo se arma de forças defensivas. Esta síndrome foi dividida em três fases: alarme ou alerta, resistência e exaustão, sendo que a fase de reação de alarme foi compreendida como a emergência na qual o organismo é exposto ao agente capaz de eliciar sua reação. Nessa fase há aumento da freqüência cardíaca e da pressão arterial, incluindo reações neuroendócrinas, há ainda, maior irrigação de sangue nos vasos, aumento de oxigenação, maior energia para os músculos, entre outras reações. A fase de resistência é a conseqüência da anterior, caso o agente agressor continue a ação. Nesta fase pode ocorrer aumento de atividade no córtex supra-renal, atrofia das estruturas linfáticas, ulcerações no aparelho digestivo, além de sintomas como irritabilidade, insônia, alterações de humor e diminuição de desejo sexual. A terceira fase chamada de exaustão é descrita como uma falha dos mecanismos adaptativos. Nas situações em que se torna possível o controle do organismo diante dos eventos estressores há o retorno à fase de alarme e na seqüência o retorno à homeostase, contudo, diante da persistência e do aumento da intensidade do estímulo estressor o organismo pode não ter sucesso em sua busca de adaptação podendo ter inúmeros prejuízos, inclusive, desenvolvimento de doenças graves.

É possível observar a ocorrência de estresse em diversas situações vividas pelo indivíduo, entre elas encontra-se destaque especial às situações relacionadas ao trabalho. Murta e Tróccoli (2004) ressaltaram que a atividade produtiva é geradora de satisfação, entretanto com a tecnicidade, os altos níveis de exigência, de controle e de pressão exercidos, são cada vez mais notáveis os reflexos negativos no trabalhador (Mallar \& Capitão, 2004; Souza, Baptista \& Xidieh, 2001).

Edwards e Cooper (1990) discutiram sobre a abordagem do estresse ocupacional envolvendo a pessoa e o ambiente, em que o estresse aconteceria quando há perda de correspondência entre as características pessoais em termos de habilidades e valores e o atendimento às demandas do ambiente. Essa relação pessoa-ambiente pode ser descrita como objetiva porque se refere às variáveis existentes e independentes de percepções individuais e, subjetiva porque as variáveis dependem da percepção do indivíduo. Ainda dentro dessa relação encontram-se duas situações que merecem destaque, pois por um lado o ambiente pode ser sentido como motivador, ao ressaltar objetivos e valores, enquanto que em contrapartida, o mesmo ambiente tem demandas que vão exigir do indivíduo tipos de habilidades e capacidades pessoais para enfrentá-las. Trata-se de um modelo complexo e mais amplo do que o exposto, 
entretanto, vale ressaltar sua importância e o destaque de que o estresse ocupacional envolve não apenas as reações do indivíduo, mas o papel do próprio ambiente enquanto demandas que são exigidas.

Mudanças ambientais são observadas desde a revolução industrial, envolvendo a natureza e a organização do trabalho. Essas mudanças propiciaram uma nova reorganização social que deu lugar especial ao trabalho na vida das pessoas, que permitiu um grande crescimento inesperado no padrão de vida, mas também expôs as pessoas a situações complexas e os riscos de acidentes eram mais freqüentes. A conseqüência menos óbvia foi a produção de grande perda de autonomia sobre o sistema laboral e a organização, o entendimento do valor e da divisão do trabalho. Neste sentido, compreender o estresse ocupacional é buscar estudar os aspectos do trabalho que estão relacionados a efeitos desagradáveis. $\mathrm{O}$ ambiente de trabalho pode favorecer ou não o conforto, diante de fatores físicos (temperatura, umidade etc.) e fatores psicológicos como expor trabalhadores em condições perigosas de trabalho e excesso de horas dispensadas às atividades laborais, dentre outras (Holt, 1982).

Com o objetivo de avaliar os índices de saúde mental e os fatores psicossociais de risco, foi realizado um estudo com 400 trabalhadores de uma universidade do interior paulista, sendo $63 \%$ da amostra feminina, $49 \%$ casados e $55 \%$ com dependentes de zero a três anos. Para isso foi utilizado o SWS Survey (Self, Work and Social) que avalia o estresse ocupacional e os fatores de risco; os estressores e os fatores de apoio nas dimensões social, pessoal e trabalho. Com estudos de adaptação e validade no Brasil, os resultados encontrados sugerem que os índices de saúde mental aumentam conforme aumentam os fatores de apoio e diminuem os fatores de estresse. Os resultados denotaram ainda, que combinado ao excesso de trabalho, ser do sexo feminino favoreceu com que este grupo apresentasse mais fatores de risco e de estresse, ao passo que o sexo masculino apresentou maiores índices de saúde mental. Mesmo diante dos benefícios que o trabalho remunerado pode representar, verificou-se que o estresse contribuiu negativamente na vida das mulheres, o que pode ter refletido além do ambiente laboral. Hipotetiza- se que, por serem atribuídas às mulheres responsabilidades da casa, da família e dos filhos, essas podem se sentirem sobrecarregadas, ocasionando conflitos familiares. (Areias \& Guimarães, 2004).

No estudo de Stacciarini e Tróccoli (2001), de investigação exploratória para levantar o que a classe de enfermeiros definia como estresse, os autores realizaram uma entrevista semi-dirigida com 33 profissionais divididos em três grupos: docentes, assistenciais e administrativos. A entrevista tinha como tema quatro blocos sendo o primeiro de dados pessoais, o segundo com informações sobre o significado do estresse, o terceiro bloco mais relacionado à problemática do estresse vivenciado e o quarto mais livre para alguma informação adicional. De maneira geral, basicamente os três grupos indicaram estressores divididos em fatores parecidos, indicando que embora o estresse seja um fenômeno individual, alguns estressores são comuns como: relações de trabalho (com pacientes, equipes), papéis dos estressores (carga emocional) e fatores intrínsecos ao trabalho (recursos inadequados, carga horária e questões salariais). Uma questão importante que foi ressaltada quando analisaram estresse em mulheres trabalhadoras diz respeito à inteface trabalho-família. O mais interessante é que esta interação ora representa dificuldades no cuidado com os filhos, casa e responsabilidades, em outros momentos funcionam como suporte e apoio para o enfrentamento do estresse.

Cooke e Rousseau (1987) já apontavam o valor significativo do suporte como moderador de impacto nos estressores relacionados ao trabalho. Em sua pesquisa com professores, observou-se que pessoas casadas possuíam saúde física e mental melhor que a saúde de pessoas solteiras e estas melhor que o índice de saúde das divorciadas e separadas. Mesmo que as relações familiares apresentassem conflitos de papéis, por exemplo, esse efeito foi contrabalanceado pelo suporte e outras contribuições vindas do parceiro (a). Neste mesmo estudo, verificou-se que nos homens, o casamento e a paternidade podem apresentar efeito positivo na auto-estima e na motivação voltada ao trabalho, pois há maior demonstração de envolvimento e identificação com a organização. Por outro lado, as mulheres trabalhadoras com filhos, foram mais afetadas pelo estresse e conflito de papéis do que as mulheres solteiras e sem filhos.

Outro fator que está relacionado ao conflito trabalho-família e tem merecido preocupação por parte da sociedade e dos estudiosos é em relação às longas horas de trabalho e a possibilidade delas terem conseqüências negativas para a família e os trabalhadores. Aliteratura demonstra que há correlação positiva entre o número de horas trabalhadas e a interferência familiar (Major, Klein \& Ehrhart, 2002; Wharton \& Blair-Loy, 2006).

Como observado anteriormente, o estresse laboral é um construto importante, presente e relevante na sociedade atual. O trabalho além de ser fonte de prazer, pode favorecer insatisfações ou dificuldades até alcançar situações de estresse, que por sua vez, pode refletir seu impacto em outras relações vivenciadas pelo trabalhador, entre elas, a família ocupa lugar de destaque por exigir demandas do indivíduo e por também sofrer com o impacto do estresse, sem contar que a recíproca pode ser verdadeira, pois a família também pode promover estresse, que será refletido em outras situações, como as do trabalho.

Diante do exposto, estudos são necessários para 
ampliar a compreensão desta inter-relação trabalhofamília. Neste sentido e diante da falta de maiores estudos sobre o suporte familiar, relacionado ao estresse laboral, a presente pesquisa visou investigar a percepção de suporte familiar e sua relação com a vulnerabilidade ao estresse laboral. A hipótese central deste estudo é que a vulnerabilidade ao estresse laboral tenha relação negativa com o suporte familiar, sugerindo que quanto menor for o nível de vulnerabilidade ao estresse no trabalho, maior deverá ser o nível do suporte familiar. Outras duas hipóteses referem-se às expectativas de relação negativa entre eventos de vida estressores e suporte familiar. Como análises secundárias, buscou-se investigar diferenças entre sexos e jornada de trabalho de acordo com o suporte familiar percebido e vulnerabilidade ao estresse no trabalho.

\section{Método}

\section{Participantes}

A coleta de dados foi realizada com 530 estudantes universitários de uma instituição particular da cidade de São Paulo. No entanto, para a análise dos dados foram selecionados para compor a amostra apenas os participantes que se declararam trabalhadores, em virtude de uma das variáveis principais desse estudo ser estresse no trabalho. Foram excluídos ainda, os participantes que não responderam acima de $10 \%$ dos instrumentos. A amostra final foi composta por 414 estudantes, com idade média de 25 anos $(\mathrm{DP}=6,27)$, sendo a mínima 17 e a máxima 54 anos; em relação ao sexo, houve predominância feminina correspondendo a 56,8\% $(n=235)$ da amostra final.

\section{Instrumentos}

Questionário de Identificação: Os participantes responderam a um questionário de identificação, contendo 24 perguntas objetivas acerca da identificação dos mesmos e outras questões pertinentes, bem como características socioeconômicas, com base no Critério de Classificação Econômica Brasil - CCEB (ANEP, 2000), além de informações sobre o tipo de trabalho e jornada semanal de horas de trabalho. A última questão condensou os eventos de vida da entrevista de Paykel, adaptada por Oliveira, Fonseca e Del Porto (2002), separados em dez categorias: trabalho, educação, questões financeiras, saúde, lutos e perdas, migração ou mudanças, namoro e coabitação, questões legais, família e matrimônio. Para esta pesquisa foi necessário converter os itens da entrevista para a forma de auto-relato, nos quais o participante assinalava os acontecimentos vividos no último ano.

Inventário de Percepção de Suporte Familiar (IPSF) (Baptista, 2005): tem o objetivo de avaliar a percepção do respondente frente ao suporte que recebe dos integrantes da sua família. $\mathrm{O}$ instrumento consta de 42 itens, com opções Likert de três pontos, divididos em três dimensões, sendo o Fator 1 denominado de Afetivo-Consistente, que envolve questões a respeito da expressão de afetividade entre os membros, seja verbal ou não, interesse, simpatia, acolhimento, consistência de comportamentos e habilidades na resolução de problemas. O segundo Fator é denominado de Adaptação Familiar e que pontuado de maneira invertida, questiona os sentimentos negativos em relação ao grupo familiar, como raiva, isolamento, exclusão, falta de compreensão entre outros, que apontam a ausência de adaptação no grupo. O último Fator é denominado Autonomia, em cujos itens se avaliam as relações de confiança, liberdade e privacidade entre os membros. A análise fatorial produzida pelo método dos componentes principais com rotação oblimim, normalizado por Kaiser, teve índice de KMO de 0,95 e o teste de esfericidade de Bartlett foi significativo ao nível de 0,00001 ( $\chi 2=17041,35 ; \mathrm{gl}=861)$, os eingenvalues desta análise permaneceram superiores a 2,5 e a variância total explicada pelo instrumento foi de 41,4\% (Baptista, 2007;2009).

Escala de Vulnerabilidade ao Estresse no Trabalho (EVENT) (Sisto, Baptista, Noronha \& Santos, 2007): O objetivo da escala é avaliar a vulnerabilidade do indivíduo frente aos estressores no ambiente de trabalho, ou seja, quanto às circunstâncias do cotidiano do trabalho influenciam a conduta da pessoa, a ponto de caracterizar certa fragilidade. As questões envolvem a percepção do indivíduo frente à instituição organizacional, à filosofia da empresa, relacionamentos interpessoais no ambiente de trabalho e de sentimentos do respondente frente às suas atividades laborais, como motivação e autonomia. É uma escala tipo Likert de três pontos, estruturado em 40 itens, contendo três fatores, descritos a seguir. Clima e Funcionamento Organizacional é o primeiro fator, e remete às situações de ambiente físico inadequado, chefia despreparada, dificuldades pessoais com a chefia, expectativa excessiva de superiores, falta de oportunidade e de ascensão no trabalho, falta de perspectiva profissional, ausência de plano de cargos e salários, de solidariedade, não ser valorizado, salário inadequado para a função, ter autoridade rejeitada pelos iguais ou superiores, além de tom autoritário de meus superiores. O segundo fator foi denominado de Pressão no Trabalho, englobando os aspectos acúmulo de funções e de trabalho, trabalhos não pertencentes à função exercida, excesso de responsabilidade nas atividades diárias, necessidade fazer o serviço de colegas, prazos para realização de trabalhos, ritmo acelerado e ter mais obrigações que demais colegas. O terceiro fator, chamado de Infra- estrutura, compreendendo os aspectos jornadas dobradas, doença ou acidente pessoal, equipamento precário, licença de saúde recorrente dos colegas, mudança no horário de trabalho, mudança de status 
financeiro, mudanças de chefia, perspectivas de promoção vinculadas à idéia de transferência, escassa cooperação da equipe para trabalhos que deveriam ser feitos em grupo, salários atrasados e problemas no ambiente físico, como iluminação.

\section{Procedimento}

Diante da aprovação da Comissão de Ética, as salas de aula foram escolhidas por conveniência e após a informação aos participantes do Termo de Consentimento Livre e Esclarecido - TCLE foi dado início à coleta de dados e aplicação dos instrumentos. A coleta de dados demorou em média 1 hora. A seqüência dos instrumentos foi a mesma em todas as aplicações: TCLE, Questionário de Identificação, IPSF e EVENT, sendo os horários de aplicação durante as aulas, no período diurno e noturno.

\section{Resultados}

Os dados do IPSF foram correlacionados com os da EVENT e os resultados encontram-se descritos a seguir. Esperava-se que os resultados da EVENT se correlacionassem negativamente com os do IPSF, isto é quanto menor for o nível de vulnerabilidade ao estresse no trabalho, maior deverá ser o nível do suporte familiar. Essa hipótese central foi confirmada, pois o EVENT total correlacionou-se negativamente com todas as dimensões do IPSF, indicando que quanto maior a vulnerabilidade ao estresse laboral, menor a percepção de suporte recebido da família em termos de afetividade, diálogo, carinho, capacidade na resolução de problemas, integração, independência, dentre outros. Esses resultados podem ser conferidos na Tabela 1.

O fator Clima e Funcionamento Organizacional correlacionou negativamente com todas as dimensões do IPSF. Esse resultado permite inferir que com o aumento dos resultados na dimensão Clima e Funcionamento Organizacional, representada pelo ambiente físico inadequado, como despreparo da chefia, falta de oportunidades no trabalho e solidariedade, diminuiriam os resultados nas dimensões IPSF Afetivo-Consistente, calcada na forma como são representadas as relações afetivas no ambiente familiar, Autonomia Familiar que representa o quanto a independência e autonomia é exercitada na família, bem como a Adaptação Familiar que se relaciona como quanto o indivíduo sente-se adaptado, aceito e querido no grupo familiar, além da dimensão Suporte Total.

As dimensões Pressão no Trabalho e Infraestrutura e Rotina correlacionaram-se apenas com Adaptação Familiar, indicando que quanto maiores sobrecargas laborais (de chefia, colegas, prazos, etc.) e os aspectos jornadas dobradas, doença ou acidente pessoal, equipamento precário, licença de saúde recorrente dos colegas, mudança no horário de trabalho, mudança de status financeiro, mudanças de chefia, dentre outras, menor foi a percepção de que o indivíduo está adaptado ao seu grupo familiar.

Esperava-se que os eventos de vida se correlacionassem negativamente com as dimensões do IPSF, de forma que quanto menos eventos de vida, maior o suporte familiar. Essa hipótese foi confirmada parcialmente (Tabela 2). Especificamente, encontraram-se correlacionados com a dimensão de Afetivo-Consistente, os eventos relacionados ao namoro/coabitação e família; e com a dimensão Adaptação Familiar, os eventos de saúde, família e eventos totais. Considerando o Suporte Familiar total, foram significativas as correlações com os eventos namoro/coabitação, família e eventos totais.

$\mathrm{Na}$ última hipótese, esperava-se que os eventos de vida se correlacionassem positivamente com as dimensões da EVENT, ou seja, quanto mais eventos de vida, maiores seriam os níveis de vulnerabilidade do estresse. De maneira geral, esta hipótese também foi confirmada, como é possível notar na Tabela 3. Correlacionaram-se com o fator Clima e Funcionamento Organizacional apenas os eventos totais; com o fator Pressão no Trabalho, os eventos relacionados às questões legais; e com a Infra -estrutura e Rotina, os eventos referentes a lutos e perdas e eventos totais; o Estresse Total

Tabela 1

Correlação de Spearman entre os resultados obtidos nos fatores do IPSF e da EVENT.

\begin{tabular}{|c|c|c|c|c|c|c|c|c|}
\hline & \multicolumn{2}{|c|}{$\begin{array}{c}\text { Clima e } \\
\text { Funcionamento } \\
\text { Organizacional }\end{array}$} & \multicolumn{2}{|c|}{ Pressão no Trabalho } & \multicolumn{2}{|c|}{ Infre-Estrutura e Rotina } & \multicolumn{2}{|c|}{ EVENT - Total } \\
\hline & $\mathrm{r}_{5}$ & $p$ & $\mathrm{r}_{\mathrm{s}}$ & $p$ & $\mathrm{r}_{5}$ & $p$ & $\mathrm{r}_{\mathrm{5}}$ & $p$ \\
\hline Afetivo-Consistente & $-0,18(* \pi)$ & 0,000 & 0,02 & 0,655 & $-0,05$ & 0,348 & $-0,12(*)$ & 0,014 \\
\hline Adaptação-famil iar & $-0,27(* \cdots)$ & 0,000 & $-0,14(* *)$ & 0,005 & $4,17(* *)$ & 0,000 & $-0,26(* \infty)$ & 0,000 \\
\hline Autonomia Familiar & $-0,17(* \cdots)$ & 0,001 & $-0,08$ & 0,121 & $-0,08$ & 0,096 & $-0,15(* \infty)$ & 0,002 \\
\hline Suporte Familiar Total & $-0,24(* \infty)$ & 0,000 & $-0,03$ & 0,498 & $-0,10$ & 0,054 & $-0,19(* *)$ & 0,000 \\
\hline
\end{tabular}


Tabela 2

Correlação de Spearman entre as categorias dos eventos de vida e os fatores do IPSF

\begin{tabular}{|c|c|c|c|c|c|c|c|c|}
\hline & \multicolumn{2}{|c|}{$\begin{array}{c}\text { Afetivo- } \\
\text { Consistente }\end{array}$} & \multicolumn{2}{|c|}{ Autonomia Familiar } & \multicolumn{2}{|c|}{$\begin{array}{c}\text { Adaptação } \\
\text { Familiar }\end{array}$} & \multicolumn{2}{|c|}{$\begin{array}{c}\text { Suporte Familiar } \\
\text { Total }\end{array}$} \\
\hline & $\mathrm{r}_{\mathrm{s}}$ & $p$ & $\mathrm{r}_{\mathrm{s}}$ & $p$ & $\mathrm{r}_{\mathrm{s}}$ & $p$ & $\mathrm{r}_{\mathrm{s}}$ & $p$ \\
\hline Trabalho & $-0,06$ & 0,210 & $-0,08$ & 0,129 & $-0,09$ & 0,086 & $-0,09$ & 0,072 \\
\hline Educação & 0,01 & 0,812 & $-0,03$ & 0,498 & $-0,10$ & 0,850 & $-0,00$ & 0,958 \\
\hline Questōes financeiras & 0,02 & 0,644 & 0,028 & 0,709 & $-0,05$ & 0,294 & 0,01 & 0,870 \\
\hline Saúde & $-0,06$ & 0,205 & $-0,08$ & 0,128 & $-0,11\left(^{*}\right)$ & 0,029 & $-0,08$ & 0,094 \\
\hline Lutos e perdas & 0,06 & 0,241 & $-0,02$ & 0,626 & 0,02 & 0,686 & 0,05 & 0,329 \\
\hline Migração/Mudança & 0,09 & 0,072 & 0,04 & 0,483 & 0,05 & 0,352 & 0,09 & 0,073 \\
\hline Namoro/Coabitação & $-0,16(* *)$ & 0,001 & $-0,10$ & 0,051 & $-0,08$ & 0,112 & $-0,14(* *)$ & 0,003 \\
\hline Questōes legais & $-0,02$ & 0,757 & 0,01 & 0,787 & $-0,05$ & 0,315 & $-0,02$ & 0,752 \\
\hline Familia & $-0,15(* *)$ & 0,002 & $-0,04$ & 0,374 & $-0,24(* *)$ & 0,000 & $-0,18(* *)$ & 0,000 \\
\hline Matrimônio & $-0,04$ & 0,407 & $-0,05$ & 0,311 & $-0,03$ & 0,502 & $-0,05$ & 0,359 \\
\hline Eventos Totais & $-0,09$ & 0,078 & $-0,09$ & 0,072 & $-0,15(* *)$ & 0,002 & $-0,12(*)$ & 0,017 \\
\hline
\end{tabular}

** Correlação é significativa ao nivel 0,01 .

* Correlação é significativa ao nivel 0,05 .

correlacionou-se com os eventos no trabalho, lutos e perdas, questões legais e eventos totais.

Como análises secundárias, foram buscadas diferenças de média entre os grupos de sexo, de acordo com os resultados nos dois instrumentos (IPSF e EVENT). Os dados encontrados demonstraram não haver diferença significativa quanto ao sexo nas dimensões da EVENT. No entanto, em relação ao sexo e o suporte familiar houve diferença significativa. A dimensão Autonomia Familiar apresentou diferença significativa quanto ao sexo ( $U=18516,5$ e $p=0,035)$. Homens $(\mathrm{M}=221,56)$ apresentaram média superior na percepção de autonomia familiar, quando comparados às mulheres $(\mathrm{M}=196,79)$.

No que tange à jornada de trabalho foram analisados os grupos que trabalham até 30 horas, de 30 a 40/44 horas e acima de 44 horas, de acordo com as respostas da EVENT. Foram encontradas diferenças significativas entre as dimensões Clima e Funcionamento Organizacional ( $\mathrm{M}=167,07$; $M=208,48 ; M=225,48$, respectivamente até as 30 horas; de 31 a 40/44 horas; e acima de 44 horas), com $\mathrm{H}=10,959$ e $\mathrm{p}=0,004$; Pressão no Trabalho ( $M=170,09 ; M=201,05 ; M=242,25$, respectivamente), com $\mathrm{H}=16,232$ e $\mathrm{p}=0,000$; e Estresse total $(\mathrm{M}=171,06$; $\mathrm{M}=201,93 ; \mathrm{M}=239,16$, respectivamente), com $\mathrm{H}=$ 14,261 e $p=0,001$. Essas relações demonstram que a vulnerabilidade ao estresse no trabalho foi maior nos grupos com maior carga horária de trabalho.

\section{Discussão}

Como apontam Stacciarini e Tróccoli (2001), a falta de reconhecimento, sensação de impotência no trabalho, problemas com função, carga horária e recursos inadequados podem contribuem para o aparecimento do estresse. Corroboram também com Mallar e Capitão (2004) e Murta e Tróccoli (2004)

Tabela 3

Correlação de Spearman entre as categorias dos eventos de vida e os fatores da EVENT.

\begin{tabular}{|c|c|c|c|c|c|c|c|c|}
\hline & \multicolumn{2}{|c|}{$\begin{array}{l}\text { Clima e Func. } \\
\text { Organizacional }\end{array}$} & \multicolumn{2}{|c|}{$\begin{array}{c}\text { Pressão no } \\
\text { trabalho }\end{array}$} & \multicolumn{2}{|c|}{$\begin{array}{c}\text { Infra-estrutura e } \\
\text { Rotina }\end{array}$} & \multicolumn{2}{|c|}{ Estresse Total } \\
\hline & $r_{s}$ & $p$ & $r_{s}$ & $p$ & $r_{s}$ & $P$ & $r_{s}$ & $P$ \\
\hline Trabalho & 0,09 & 0,082 & 0,09 & 0,064 & 0,05 & 0,341 & $0,11\left(^{*}\right)$ & 0,026 \\
\hline Educação & $-0,02$ & 0,745 & $-0,02$ & 0,632 & 0,07 & 0,143 & 0,01 & 0,924 \\
\hline Questões financeiras & 0,07 & 0,173 & 0,07 & 0,166 & 0,02 & 0,716 & 0,06 & 0,197 \\
\hline Saúde & 0,05 & 0,298 & $-0,00$ & 0,938 & 0,05 & 0,334 & 0,04 & 0,413 \\
\hline Lutos e perdas & 0,08 & 0,101 & 0,08 & 0,095 & $0,14(* *)$ & 0,005 & $0,11(*)$ & 0,027 \\
\hline Migração/Mudança & $-0,03$ & 0,543 & $-0,07$ & 0,157 & 0,03 & 0,572 & $-0,02$ & 0,642 \\
\hline Namoro / Coabitação & 0,08 & 0,105 & 0,02 & 0,713 & 0,05 & 0,323 & 0,06 & 0,192 \\
\hline Quest ōes legais & 0,07 & 0,159 & $0,12(*)$ & 0,012 & 0,09 & 0,076 & $0,10(*)$ & 0,034 \\
\hline Família & 0,01 & 0,808 & 0,06 & 0,222 & 0,08 & 0,128 & 0,06 & 0,235 \\
\hline Matrimônio & 0,04 & 0,410 & 0,02 & 0,742 & 0,07 & 0,141 & 0,05 & 0,272 \\
\hline Eventos Totais & $0,11\left(^{*}\right)$ & 0,024 & 0,09 & 0,065 & $0,13(*)$ & 0,011 & $0,14(* *)$ & 0,006 \\
\hline
\end{tabular}


quanto aos apontamentos de que o desgaste da organização aumenta o potencial para gerar conflitos e de que a pressão no trabalho e situações de segurança tornam as pessoas mais vulneráveis, pois exigem do trabalhador habilidades para enfrentar essas situações.

Verificou-se que quanto maior foi o nível de vulnerabilidade ao estresse menor o nível de suporte familiar percebido em dez das dezesseis possibilidades de correlação entre os instrumentos. Assim como encontrado, o suporte familiar pode favorecer níveis de saúde e proteção o indivíduo frente às situações estressantes, enquanto que a falta de suporte contribui para o aumento da vulnerabilidade (Castro \& cols., 1997). Alchaer e colabores (1994) já destacaram que indivíduos com maiores níveis de estresse apresentavam maiores níveis de disfunção familiar e Baptista $(2005,2007)$ ressaltou o papel do suporte familiar como amortecedor dos efeitos estressantes.

As correlações encontradas entre a dimensão Afetivo-Consistente e os eventos de Namoro/ Coabitação e Família, indicaram que a falta interesse afetivo pelos outros, distanciamento ou quando o ambiente não permite expressar o afeto, tampouco uma comunicação assertiva, as áreas de Namoro/ Coabitação e Familiar são sentidas como estressantes, pois nestas duas, as relações afetivas são muito importantes, principalmente porque segundo os relatos dos participantes, os eventos vividos, envolveram situações de rompimentos afetivos em relações importantes (namoro e/ou noivado), problemas com familiares e conflitos de adaptação na família.

A dimensão Adaptação Familiar correlacionou negativamente com as dimensões Saúde, Família e Total dos Eventos, sugerindo que não se sentir adaptado, compreendido e possuir sentimentos de vergonha, irritação e perceber as relações afetivas como agressivas, encontra-se relacionado nesta amostra a problemas de saúde vivenciados e situações estressantes no âmbito familiar, tais como, conflito entre os membros, mudanças na rotina e adaptação às novas demandas. De fato, quando o indivíduo sente que pertence a um grupo que o aceita e está disponível para apoiá-lo, isso se torna um fator de proteção contra situações estressantes. A dimensão Suporte Familiar Total, correlacionou-se com as áreas de namoro, família e eventos totais, confirmando os dados encontrados. Estes resultados corroboram os achados em que os eventos de vida agem como estressores que interagem com o nível de saúde mental e na ausência de fatores protetivos presentes no suporte e a rede social, podem ser agravados, uma vez que receber suporte pode ser fundamental nas situações de enfrentamento (Alchaer \& cols., 1994).

As correlações positivas encontradas entre os Eventos de Vida e a Escala de Vulnerabilidade ao Estresse, indicaram que quanto mais eventos de vida ocorrem, maior é a vulnerabilidade ao estresse laboral. Selye (1982) já discutia a importância do papel dos estressores, afirmando que a quantidade de eventos estressores pode minar a capacidade de enfrentamento de situações pelo indivíduo. Edwards e Cooper (1990) consideraram que o estresse ocupacional envolve pessoa e ambiente e, este ocorre quando não há correspondência entre as características pessoais (habilidades e valores) no atendimento à demanda.

Ainda em correspondente à correlação entre eventos de vida e vulnerabilidade ao estresse no trabalho, as questões de Lutos envolveram desde mortes de parentes e amigos próximos a perdas de objetos de valor, o que denota fragilidade diante desta situação, vulnerabilidade associada à falta de estrutura da organização com as condições de trabalho, como excesso de jornada, equipamento precário, mudanças de status financeiro, de setor, que podem refletir em instabilidade no ambiente organizacional.

Sobre as análises secundárias, os resultados encontrados demonstraram não haver diferença significativa quanto ao sexo nas dimensões da EVENT. Esses resultados divergem dos de Areias e Guimarães (2004) que indicaram que as mulheres apresentaram níveis maiores de estresse do que os homens, principalmente porque mulheres desempenhariam dupla jornada. Quanto ao suporte familiar, as mulheres perceberam o suporte familiar como menor em termos de autonomia. A este resultado é atribuída a questão cultural, pois as mulheres seriam mais vulneráveis à dinâmica familiar, pela educação que receberam, em uma sociedade "machista", enquanto que os homens são mais estimulados a serem independentes.

A análise envolvendo jornada de trabalho indicou que menos tempo de trabalho na organização, diminuem as sensações desagradáveis que envolvem cobranças, falta de suporte e apoio, prazos apertados e responsabilidade excessiva. Wharton e Blair-Loy (2006), ressaltaram o fato de que longas horas de trabalho estão associadas com aumento de estresse. Major e colaboradores (2002) reforçaram que as horas devotadas ao trabalho, além do estresse causado pela sobrecarga, dificultam o tempo disponível para outras atividades, inclusive o tempo a ser devotado à família. É necessário destacar que essa pesquisa tem suas limitações, devendo ser aplicada inclusive para outras amostras, talvez se controlando áreas de atuação (comércio, indústria, prestação de serviço), função, idade, constituição familiar, dentre outras variáveis.

\section{Referências}

Ackerman, N. W. (1978). Diagnóstico y tratamiento de las relaciones familiares: Psicodinamismos de la vida familiar. $\left(6^{\mathrm{a}}\right.$. ed.). Buenos Aires: Hormé

Alchaer, J. R., Bahsas, F., Nieto, R. H., \& Salinas, P. J. (1994). Relación entre el funcionalismo familiar: el estrés y la ansiedad. Medula, 3(3/4) 81-6.

Areias, M. E. Q., \& Guimarães, L. A. M. (2004) Gênero e estresse em trabalhadores de uma universidade pública do estado 
de São Paulo. Psicologia em Estudo, 9(2): 255-262.

Associação Nacional de Empresas de Pesquisa - ANEP (2000). Critério de Classificação

Econômica Brasil - CCEB. Disponível em: www.anep.org.br. Acesso em: 23 mar. 05.

Baptista, M. N. (2005). Desenvolvimento do Inventário de Percepção de Suporte Familiar (IPSF): estudos psicométricos preliminares. Psico-USF, 10(1), 11-19.

Baptista, M. N. (2007). Inventário de Percepção de Suporte Familiar (IPSF): estudo

componencial em duas configurações. Psicologia: Ciência e Profissão, 27(3). Disponível em: http://pepsic.homolog.bvsalud. org/scielo.php?pid=S1414-98932007000300010\&script $=$ sci_ arttext. Acesso em: 20 out. 2010.

Baptista, M. N. (2009). Inventário de Percepção de Suporte Familiar - IPSF (Manual). São Paulo: Vetor Editora.

Baptista, M. N., Baptista, A. S. D., \& Dias, R. R. (2001). Estrutura e Suporte Familiar como Fatores de Risco na Depressão de Adolescentes. Psicologia Ciência e Profissão, 21(2), 52-61.

Baum, A., \& Posluszny, D. M. (1999). Health Psychology: Mapping Biobehavioral Contributions to Health and Illness. Annual Review of Psychology, 50, 137-163.

Caldas, C. P. (2003). Envelhecimento com dependência: responsabilidades e demandas da família. Rio de Janeiro: Cadernos de Saúde Pública, 19(3): 773-781.

Calnan, M., Wadsworth, E., May, M., Smith, A., \& Wainwright, D. (2004). Job strain, effort - reward imbalance, and stress at work: competing or complementary models? Scandinavian Journal of Public Health_32, 84-93.

Campos, E. P. (2004). Suporte Social e Família. Em: J. Mello Filho, \& M. Burd (orgs).

Doença e Família. São Paulo: Casa do Psicólogo.

Carvalho, I. M. M., \& Almeida, P. H. (2003). Família e Proteção Social. São Paulo em

Perspectiva, 17(2), 109-122.

Castro, R., Campero, L., \& Hernández, B. (1997). La investigación sobre apoyo social en salud: situación actual y nuevos desafíos. Revista de Saúde Pública, 31(4), 425-435.

Cooke, R. A., \& Rousseau, D. M. (1984). Stress and Strain from Family Roles and Work-Role Expectations. Journal of Applied Psychology, 69(2), 252-260.

Cooke, B. D., Rossmann, M. M., \& McCubbin, H. I., \& Patterson, J. M. (1988). Examining

the Definition and Assessment of Social Support: A Resource for Individuals and

Families. Family Relations, 37, 221-216.

Edwards, J. R., Cooper, C. L. (1990). The person-environment fit approach to stress:

recurring problems and some suggested solutions. Journal of Organizational Behavior

11, 293-307.

Féres-Carneiro, T. (1983). Família: Diagnóstico e Terapia. Rio de Janeiro: Zahar.

Holt, R. R. (1982). Occupational Stress. In: Goldberg, L \& Brenznit, S. Handbook of Stress: Theoretical and Clinical Aspects. The Fress Press: New York.
Major, V. S., Klein, K. J., \& Ehrhart, M. G. (2002). Work time, Work Interference With

family, and Psychological distress. Journal of Applied Psychology 87(30), 427-436.

Mallar, S. C., \& Capitão, C. G. (2004). Burnout e hardiness: um estudo de evidência de

validade. Psico-USF, 9(1), 19-29.

Mota, E. L. A, Franco, A. L. S., \& Motta, M. C. (1999). Migração, estresse e fatores

psicossociais na determinação da saúde da criança. Psicologia:

Reflexão e Crítica, 12(1), 119-

132.

Murta, S. G., \& Tróccoli, B. T. (2004). Avaliação de intervenção em estresse ocupacional. Psicologia Teoria e Pesquisa, 20(1), 39 47.

Myers, H. L., \& Myers L. B. (2004). A Study of the work stress and health of GDPs. British Dental Journal, 197(2), 83.

Oliveira, M. G., Fonseca, P. P., \& Del Porto, J. A. (2002). Versão brasileira da entrevista de Paykel para eventos de vida recentes. Revista Brasileira de Terapia Comportamental e Cognitiva, IV(1), 47-61.

Organización Panamericana de La Salud [OPS] (2003). $44^{\circ}$ Consejo directivo e $55^{a}$ Sesión del Comité Regional. Washington.

Pearlin, L. I. (1982). The Social Contexts of Stress. Em: L. Goldberg \& S. Brenznit.

Handbook of Stress: Theoretical and Clinical Aspects. The Fress Press: New York.

Salmond, S., \& Ropis, P. E. (2005). Job Stress and General Well-being: A Comparative

Study of Medical-Surgical and Home Care Nurses. Medsurg Nursing, 14(5), 301-309.

Selye, H. (1982). History and Present Status of the Stress Concept. Em: L. Goldberg \& S. Brenznit. Handbook of Stress: Theoretical and Clinical Aspects. The Fress Press: New York.

Sisto, F. F., Baptista, M. N., Noronha, A. P. \& Santos, A. A. A. (2006). Escala de

Vulnerabilidade ao Estresse no Trabalho - EVENT. Manual Técnico.

Souza, F. G., Baptista, M. N., \& Xidieh (2001). Burnout: definição e características. Infanto Rev. Neuropsiquiatria da Infância e Adolescência, 9(3), 100-102.

Stacciarini, J. M. R. \& Trócolli, B. T. (2001). O Estresse na

Atividade Ocupacional do

Enfermeiro. Revista Latino americana de Enfermagem, 9(2), $17-25$.

Wharton, A. S. \& Blair-Loy, M. (2006). Long Work Hours and Family Life. Journal of

Family Issues, 27(3) 415-436.

Recebido: $24 / 12 / 2010$

Última Revisão: 24/06/2011 Aceite Final: 09/08/2011

Sobre os autores:

Regiane Ribeiro de Aquino - Docente da Universidade Cruzeiro do Sul. Mestre em Psicologia pelo Programa de Pós Graduação em Psicologia da Universidade São Francisco, Itatiba, São Paulo.

Makilim Nunes Baptista - Doutor pelo departamento de Psiquiatria e Psicologia Médica da UNIFESP. Docente do Programa de Pós-Graduação em Psicologia da Universidade São Francisco, Itatiba, São Paulo. Bolsista de Produtividade em Pesquisa do CNPq - Nível 1D. Email: makilim.baptista@saofrancisco.edu.br.

Mayra Silva de Souza - Psicóloga. Doutora em Psicologia pelo Programa de Pós Graduação Stricto Sensu em Psicologia da Universidade São Francisco. 